\title{
EDITORIAL \\ Endoscopic synostosis surgery: case closed. Or open? Or in between?
}

\author{
Matthew D. Smyth, MD \\ Department of Neurological Surgery, Washington University, St. Louis Children's Hospital, St. Louis, Missouri
}

$\mathrm{I}$ $\mathrm{T}$ was with great interest that I reviewed the case series from the Rady Children's Hospital craniofacial center summarizing their experience with minimally invasive techniques for the management of craniosynostosis. ${ }^{2}$ This group was one of the earliest adopters and advocates of these techniques. As such, this report represents one of the largest published longitudinal series and is a meaningful contribution to the ongoing "open versus endoscopic" debate. Dalle Ore et al. report a noncontrolled retrospective chart review of 235 infants undergoing endoscopic synostosis procedures between 2000 and 2015 by a single neurosurgeon at a single institution, with the primary goal of evaluating complication and reoperation rates. The authors report no deaths or neurological injuries, with overall low perioperative complication and readmission rates. They note a higher revision rate for coronal and metopic synostosis compared with sagittal synostosis and seem to suggest that open techniques may be preferable for these two synostosis types, offering them only to highly motivated families intent on less invasive options. With the goal of an excellent aesthetic result being paramount, this is a laudable approach; each center should strive to utilize their experience and skill to optimize their outcomes for their patients. As with most of our field's published surgical case series, meaningful data on anthropomorphic/ aesthetic and neurocognitive outcomes are lacking. In that respect, this is yet another study using only cephalic index as a measure of outcome for sagittal synostosis (and no anthropomorphic measurements for metopic/coronal synostosis), with a relatively short follow-up period of 2.8 years.

Except for the two patients with lambdoid synostosis, the remaining patients underwent more extensive bone removal and osteotomies (with multiple scalp incisions) than the smaller, simple strip suturectomies typically performed at many centers. ${ }^{1,6,12}$ In sagittal synostosis, for example, the authors describe two 3-4-cm scalp incisions, a vertex strip craniectomy, bilateral parietal barrel staves, and bilateral occipital osteotomies. At our center, we abandoned the bilateral parietal wedge osteotomies after a prospective comparison showed no additional benefit ${ }^{3}$ beyond simple narrow strip craniectomies, and others have investigated this issue as well. ${ }^{16}$ For metopic and coronal synostosis, the authors are performing tarsal incisions in addition to the scalp incisions, and performing frontoorbital releases in addition to simple suturectomy. They discussed early disappointing results with suturectomy alone, and moved to a hybrid approach with more extensive bone releases and additional scalp incisions to obtain more correction at the time of surgery. Our center also experienced a learning curve early on with some early disappointing results using simple suturectomy/orthotics, but we focused on improving helmeting methods in close partnership with our orthotists and now achieve satisfactory long-term results for all the major sutures, utilizing helmets until about 12 months of age. . $, 10,17$

The Rady approach to transfusions and their discussion about the risk/benefit ratio for transfusion with contemporary screened blood products was thought provoking and has merit, although it would appear that a number of potentially unnecessary transfusions are being performed using their protocol. With an excellent average estimated blood loss (EBL) of only $25 \mathrm{ml}$ for sagittal synostoses, why should the transfusion rate approach $50 \%$ ? It seems that they are giving a relatively small volume of blood products back to balance a relatively small blood loss and exposing a large number of infants to potential transfusion risks. With the use of a narrow strip technique, the transfusion rate at our institution is less than $5 \%$ for sagittal synostosis, although in every case we have blood products ready if needed (we have had two instances of sagittal sinus-related blood loss necessitating rapid intraoperative transfusion in about 300 endoscopic operations to date). We too have observed higher EBL in metopic synostosis due to the thicker diploic bone and bridging diploic veins, 
but still manage to keep transfusions under the $10 \%$ level for patients with metopic synostosis. The Rady series has the highest transfusion rates, across the board, of any published series for endoscopic synostosis surgery, but as they report, transfusion-related complications were rare and benign. At our center, we adopt a policy of liberal use of blood products for our open calvarial vault and frontoorbital advancement (FOA) procedures, and have also observed very few transfusion-related complications. For the minimally invasive endoscopically assisted single-suture patients we believe that transfusions can be safely avoided most of the time. ${ }^{5}$

These authors reported a relatively high proportion of patients with sagittal synostosis $(81 \%)$, but much fewer with metopic (14\%) or coronal (5\%). I suspect this reflects their bias towards open or hybrid approaches for metopic and coronal synostosis. It would be interesting to know their center's overall surgical volumes for both open and endoscopic surgeries for the various synostoses over the same time and I would encourage them to consider submitting a report summarizing their total surgical volumes during this period.

The group reported that their protocol remained consistent between 2000 and 2015, other than their addition of frontoorbital releases for metopic and coronal synostosis. Could there be room for modification and evolution of their protocol? Is there really a need for three hematocrit levels postoperatively? Why did they use mannitol with Foley catheters for a 1-hour extradural surgery? Has the precordial Doppler ultrasonography actually been helpful in detecting venous air embolism (they reported no instances)? Over time at our own center we have worked to streamline our protocol and shed unnecessary monitors, interventions, and laboratory tests, ${ }^{9}$ which can help with healthcare associated costs. ${ }^{14}$

The decision to convert two of the patients from endoscopic to open (1 coronal, 1 metopic) was based on "the craniofacial surgeon's intraoperative perception of a likely suboptimal future aesthetic result." This is perplexing, because with suture release, radial brain growth over time, and good orthotic therapy, one might expect a good result regardless of the intraoperative findings. If the craniofacial surgeon was reluctant to trust the endoscopic/orthotic approach in the first place, then perhaps a planned FOA at the optimal age should have been performed.

Excluding the single patient who developed secondary coronal synostosis, 6 patients underwent second operations for "suboptimal aesthetics": 3 metopic, 1 coronal, 1 sagittal, and 1 lambdoid synostosis. Based on these results, with a small number of metopic and coronal cases, the authors suggest that they discourage the use of endoscopic techniques (including even their more aggressive tarsal incisions and frontoorbital releases) in metopic and coronal cases, offering them only to "extremely motivated" families. However, what is the reoperation rate for suboptimal aesthetic outcome using open techniques for metopic and coronal synostosis at their institution? Many large series report revision rates of $10 \%-15 \%$ using open techniques for metopic and coronal synostoses, ${ }^{4,11}$ and the aesthetic outcome may worsen over time after FOA. ${ }^{15}$ The group in Boston has published data suggesting superiority of en- doscopic release for unicoronal synostosis over traditional FOA with respect to aesthetic and ophthalmologic outcome. ${ }^{7,13}$ The authors of this study appear to suggest that the higher revision rate for coronal and metopic synostosis compared with sagittal endoscopic repair makes it a less favorable option and guide families toward larger open frontoorbital operations. I would argue that the use of an early endoscopic technique allows the majority of infants to avoid a larger open procedure despite the somewhat higher revision rate for the anterior synostoses. Paradoxically, they report a 50\% reoperation rate in lambdoid and a $1 \%$ reoperation rate in sagittal cases, but do not discourage the use of endoscopic techniques in lambdoid synostosis. At what age did the revision surgeries occur? Was there adequate time for helmeting? Normalization of skull base morphology occurs over years after endoscopic release. If a second, open procedure is offered and performed too soon after an endoscopic release it may have not been ultimately necessary. Their relatively short helmet times (5 months) may have played a role.

Their unique approach to these single-suture, nonsyndromic cases is thought provoking and raises a number of interesting questions. The addition of multiple scalp and periorbital incisions, more extensive bone removal and osteotomies, and liberal use of blood products, along with relatively short helmet durations, sets their center apart, to some extent, from centers offering minimal suturectomy with helmet therapy, smaller access incision lengths, and less "bone work." Without any outcome data provided regarding aesthetic result for the two anterior synostoses, the generalizability of their results for coronal and metopic sutures is limited. Ultimately, however, it appears that satisfactory outcomes can be achieved with a thoughtful and careful approach to these patients using less invasive techniques that reduce operative times, length of stay, healthcare costs, and discomfort for the children. Endoscopically assisted synostosis repair and its variations are clearly here to stay. Case closed. Or open? Or in between? https://thejns.org/doi/abs/10.3171/2018.3.PEDS18122

\section{References}

1. Berry-Candelario J, Ridgway EB, Grondin RT, Rogers GF, Proctor MR: Endoscope-assisted strip craniectomy and postoperative helmet therapy for treatment of craniosynostosis. Neurosurg Focus 31(2):E5, 2011

2. Dalle Ore CL, Dilip M, Brandel MG, McIntyre JK, Hoshide $\mathrm{R}$, Calayag M, et al: Endosocopic surgery for nonsyndromic craniosynostosis: a 16-year single-center experience. J

Neurosurg Pediatr [epub ahead of print July 6, 2018. DOI: 10.3171/2018.2.PEDS17364]

3. Dlouhy BJ, Nguyen DC, Patel KB, Hoben GM, Skolnick GB, Naidoo SD, et al: Endoscope-assisted management of sagittal synostosis: wide vertex suturectomy and barrel stave osteotomies versus narrow vertex suturectomy. J Neurosurg Pediatr 25:674-678, 2016

4. Engel M, Castrillon-Oberndorfer G, Hoffmann J, Muhling J, Seeberger R, Freudlsperger C: Long-term results in nonsyndromatic unilateral coronal synostosis treated with frontoorbital advancement. J Craniomaxillofac Surg 41:747-754, 2013

5. Han RH, Nguyen DC, Bruck BS, Skolnick GB, Yarbrough $\mathrm{CK}$, Naidoo SD, et al: Characterization of complications associated with open and endoscopic craniosynostosis surgery 
at a single institution. J Neurosurg Pediatr 17:361-370, 2016

6. Jimenez DF, Barone CM: Early treatment of coronal synostosis with endoscopy-assisted craniectomy and postoperative cranial orthosis therapy: 16-year experience. J Neurosurg Pediatr 12:207-219, 2013

7. MacKinnon S, Rogers GF, Gregas M, Proctor MR, Mulliken JB, Dagi LR: Treatment of unilateral coronal synostosis by endoscopic strip craniectomy or fronto-orbital advancement: ophthalmologic findings. J AAPOS 13:155-160, 2009

8. Masserano B, Woo AS, Skolnick GB, Naidoo SD, Proctor MR, Smyth MD, et al: The temporal region in unilateral coronal craniosynostosis: fronto-orbital advancement versus endoscopy-assisted strip craniectomy. Cleft Palate Craniofac J 55:423-429, 2018

9. Nguyen DC, Farber SJ, Skolnick GB, Naidoo SD, Smyth MD, Kane AA, et al: One hundred consecutive endoscopic repairs of sagittal craniosynostosis: an evolution in care. J Neurosurg Pediatr 20:410-418, 2017

10. Nguyen DC, Patel KB, Skolnick GB, Naidoo SD, Huang AH, Smyth MD, et al: Are endoscopic and open treatments of metopic synostosis equivalent in treating trigonocephaly and hypotelorism? J Craniofac Surg 26:129-134, 2015

11. Seruya M, Oh AK, Boyajian MJ, Posnick JC, Myseros JS, Yaun AL, et al: Long-term outcomes of primary craniofacial reconstruction for craniosynostosis: a 12-year experience. Plast Reconstr Surg 127:2397-2406, 2011

12. Shah MN, Kane AA, Petersen JD, Woo AS, Naidoo SD, Smyth MD: Endoscopically assisted versus open repair of sagittal craniosynostosis: the St. Louis Children's Hospital experience. J Neurosurg Pediatr 8:165-170, 2011

13. Tan SP, Proctor MR, Mulliken JB, Rogers GF: Early frontofacial symmetry after correction of unilateral coronal synostosis: frontoorbital advancement vs endoscopic strip craniectomy and helmet therapy. J Craniofac Surg 24:1190-1194, 2013

14. Vogel TW, Woo AS, Kane AA, Patel KB, Naidoo SD, Smyth MD: A comparison of costs associated with endoscopeassisted craniectomy versus open cranial vault repair for infants with sagittal synostosis. J Neurosurg Pediatr 13:324-331, 2014

15. Wes AM, Paliga JT, Goldstein JA, Whitaker LA, Bartlett SP, Taylor JA: An evaluation of complications, revisions, and long-term aesthetic outcomes in nonsyndromic metopic craniosynostosis. Plast Reconstr Surg 133:1453-1464, 2014

16. Wood BC, Ahn ES, Wang JY, Oh AK, Keating RF, Rogers GF, et al: Less is more: does the addition of barrel staves improve results in endoscopic strip craniectomy for sagittal craniosynostosis? J Neurosurg Pediatr 20:86-90, 2017

17. Zubovic E, Woo AS, Skolnick GB, Naidoo SD, Smyth MD, Patel KB: Cranial base and posterior cranial vault asymmetry after open and endoscopic repair of isolated lambdoid craniosynostosis. J Craniofac Surg 26:1568-1573, 2015

\section{Disclosures}

The author reports no conflict of interest.

\section{Response}

\section{Hal S. Meltzer, MD, ${ }^{1,3}$ and Steven R. Cohen, MD²}

1'Department of Neurosurgery, University of California San Diego; and Divisions of ${ }^{2}$ Plastic Surgery and ${ }^{3}$ Pediatric Neurosurgery, Rady Children's Hospital San Diego, California

We thank Dr. Smyth for his thoughtful review of our study and the questions he raises in his careful analysis. As advocates for the efficacy of minimally invasive ap- proaches in the treatment of craniosynostosis, we are cognizant of the arguments used by those who oppose these procedures, which typically fall into two general categories: 1) aesthetic concerns, and 2) complication concerns. ${ }^{4}$ While impressed by the reports utilizing increasingly minimal, suturectomy-alone techniques and the excellent outcomes described, we advise caution to not potentially overuse this approach. Dr. Smyth references two intriguing studies which suggest that a sagittal suturectomy alone is overall superior to the addition of bilateral anterior and posterior parietal osteotomies in the endoscopic treatment of sagittal craniosynostosis., ${ }^{3,12}$ Both studies, as ours does, utilize cranial indices in the aesthetic comparison of these treatment groups. As qualified in our paper, this metric does not reliably describe the frontal bossing and occipital tapering frequently associated with this deformity. At the international scientific meeting where one of these studies was initially presented, this proposed deficiency figured prominently in the discussion that followed. ${ }^{5}$ In regard to metopic and coronal craniosynostosis, endoscopic suturectomy followed by cranial banding has been advocated as yielding equivalent or even superior results to traditional FOA.$^{8,9}$ The average age at endoscopic treatment for the metopic and coronal patients in these studies was 3.3 and 2 months, respectively, which, especially for the coronal patients, was markedly younger than the average age at surgery of the children in our study (4.2 and 5.8 months, respectively). We have had the opportunity to care for a child who previously underwent endoscopic metopic suturectomy at another institution (Fig. 1). The operative time was brief, no transfusion was required, and the child went home the day after surgery. It would be inappropriate to judge the merits of endoscopic surgery based on this one poor aesthetic outcome, which could be due to a variety of reasons, such as marked severity of initial deformity, advanced age at initial surgery, inadequate bone removal, delay to initiation of cranial banding, inadequate cranial banding, etc. However, if we hope to convince others of the reconstructive value of these innovative surgical approaches, it may be more helpful to focus on the identification of ideal candidates and care nuances for endoscopic procedures (very young especially if skull base involved, prolonged cranial banding especially if older or if skull base involved, milder rather than more severe initial deformities especially in older children, etc.) rather than to emphasize that these procedures may be a priori equivalent or superior to traditional open approaches. Suturectomy alone fell out of favor in the past and we should be careful not to oversell this approach in the future. ${ }^{6}$

In our experience to date, we have managed to avoid significant endoscopic treatment-related morbidity, but there have been allegations that overall, severe complications have been underreported in published experiences. ${ }^{4}$ At least 1 treatment-associated death not primarily reported is alleged to have occurred and we have described 1 referred case of repair of a postoperative growing skull fracture equivalent likely related to an unrecognized dural tear at the initial procedure. ${ }^{1,4}$ As experience in these procedures accrues, we encourage the sharing of additional treatment-related complications to alert others to their existence, help minimize future occurrences, and discour- 


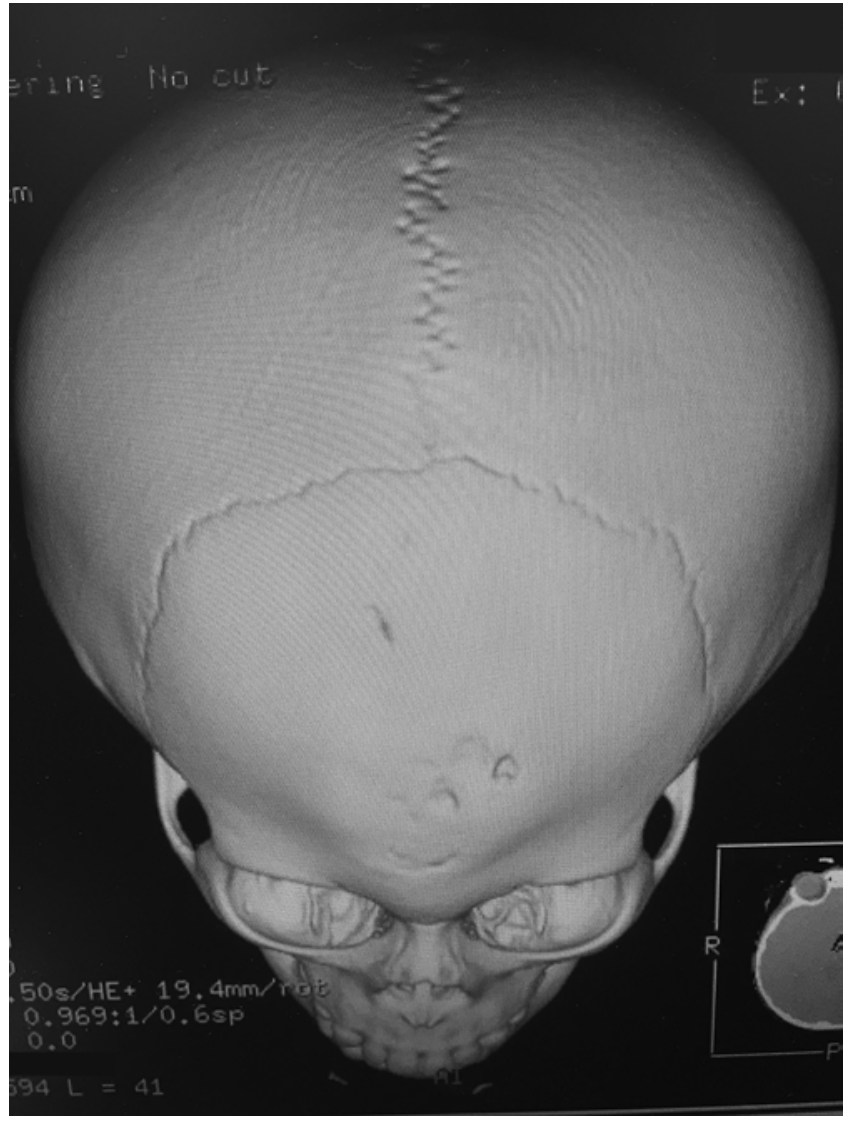

FIG. 1. Postoperative 3D CT scan after endoscopic metopic suturectomy.

age notions of unrealistic perfection. Regarding some of our practices meant to limit treatment-related morbidity, Dr. Smyth accurately observes that given the low average blood loss we report with endoscopic sagittal synostosis repair, a $50 \%$ overall transfusion rate is likely unjustifiably high. Almost $80 \%$ of these transfusions were solely intraoperative, and at our institution, typically made at the discretion of the attending anesthesiologist. Given the data reported by craniofacial centers of excellence such as Dr. Smyth's and others, we as a specialty can help educate our colleagues in understanding the expected risk of significant intraoperative hemorrhage in endoscopic versus traditional reconstructive procedures, and the likely adequacy of having blood products readily available to transfuse as needed, rather than have them proactively transfused and sometimes unneeded. ${ }^{2,7}$ However, $20 \%$ of our transfused patients were transfused postoperatively when our threshold hematocrit level of 21 was reached, and in this regard, we found the trend in hematocrit levels immediately postoperatively, later in the evening, and early the next morning very helpful in these postoperative transfusion decisions. We also find the use of a mannitol bolus and the temporary lowering of pCO2 intraoperatively facilitates the maintenance of epidural corridors during surgery and minimizes associated dural retraction. Additionally, while we wholeheartedly support efforts to optimize operative economic efficiencies and have not observed a case of venous air embolism to date, a rate of almost $10 \%$ has been previously reported during endoscopic procedures, and therefore we are not comfortable with discontinuing the use of the noninvasive precordial Doppler ultrasonography monitoring at this time. ${ }^{10}$

We are grateful for the opportunity to share our experience in the endoscopic treatment of nonsyndromic craniosynostosis with the readership of the Journal of Neurosurgery: Pediatrics and appreciate the insightful comments of Dr. Smyth and his colleagues on the Editorial Board. As we approach the 25th anniversary of the first descriptions of these innovative approaches, we reflect on the totality of the published experiences with them to date and the question Dr. Smyth poses. ${ }^{11}$ These endoscopic procedures are certainly here to stay as we strive to understand, as with all surgical procedures, their optimal indications and the perioperative nuances associated with their optimal efficacy and safety.

\section{References}

1. Aryan HE, Meltzer HS, Gerras G, Jandial R, Levy ML. Leptomeningeal cyst development after endoscopic craniosynostosis repair. Neurosurgery 55:235-238, 2004

2. Berry-Candelario J, Ridgway EB, Grondin RT, Rogers GF, Proctor MR: Endoscope-assisted strip craniectomy and postoperative helmet therapy for treatment of craniosynostosis. Neurosurg Focus 31(2):E5, 2011

3. Dlouhy BJ, Nguyen DC, Patel KB, Hoben GM, Skolnick GB, Naidoo SD, et al: Endoscope-assisted management of sagittal synostosis: wide vertex suturectomy and barrel stave osteotomies versus narrow vertex suturectomy. J Neurosurg Pediatr 25:674-678, 2016

4. Kung TA, Vercler CJ, Muraszko KM, Buchman SR: Endoscopic strip craniectomy for craniosynostosis: do we really understand the indications, outcomes, and risks? J Craniofac Surg 27:293-298, 2016

5. Magge SN, Wood BC, Ahn ES, Wang JY, Keating RF, Rogers GF: Does the addition of barrel staves improve results in endoscopic strip craniectomy for sagittal craniosynostosis?, in 44th Annual Meeting of the International Society for Pediatric Neurosurgery. Kobe, Japan: ISPN, 2016

6. Mehta VA, Bettagowda C, Jallo GI, Ahn ES: The evolution of surgical management for craniosynostosis. Neurosurg Focus 29(6):E5, 2010

7. Nguyen DC, Farber SJ, Skolnick GB, Naidoo SD, Smyth MD, Kane AA, et al: One hundred consecutive endoscopic repairs of sagittal craniosynostosis: an evolution in care. $\mathbf{J}$ Neurosurg Pediatr 20:410-418, 2017

8. Nguyen DC, Patel KB, Skolnick GB, Naidoo SD, Huang AH, Smyth MD, et al: Are endoscopic and open treatments of metopic synostosis equivalent in treating trigonocephaly and hypotelorism? J Craniofac Surg 26:129-134, 2015

9. Tan SP, Proctor MR, Mulliken JB, Rogers GF: Early frontofacial symmetry after correction of unilateral coronal synostosis: frontoorbital advancement vs endoscopic strip craniectomy and helmet therapy. J Craniofac Surg 24:1190-1194, 2013

10. Tobias JD, Johnson JO, Jimenez DF, Barone CM, McBride DS Jr: Venous air embolism during endoscopic strip craniectomy for repair of craniosynostosis in infants. Anesthesiology 95:340-342, 2001

11. Vicari F: Endoscopic correction of sagittal craniosynostosis, in Meeting of American Society of Plastic Surgeons. San Diego: ASPS, 1994

12. Wood BC, Ahn ES, Wang JY, Oh AK, Keating RF, Rogers GF, et al: Less is more: does the addition of barrel staves improve results in endoscopic strip craniectomy for sagittal craniosynostosis? J Neurosurg Pediatr 20:86-90, 2017 